\title{
Satisfacción marital en pacientes oncológicos: su relación con los eventos hirientes y la calidez brindada por su pareja
}

\author{
Marital satisfaction in oncology patients: It's relationship \\ with hurtful events and the warmth provided by their partners
}

\author{
Luisa Fernanda Granillo-Velasco y Rozzana Sánchez-Aragón \\ Universidad Nacional Autónoma de México $^{1}$
}

Autor para correspondencia: Rozzana Sánchez Aragón, rozzara@unam.mx.

\begin{abstract}
RESUMEN
El cáncer afecta considerablemente la calidad de vida y la salud de quienes lo padecen, así como las de sus parejas. Al ser la pareja un vínculo significativo para las personas, la experiencia de sufrir eventos hirientes $(\mathrm{EH})$ o de calidez durante la interacción cotidiana puede causar satisfacción e insatisfacción entre sus miembros. Así, tanto las percepciones y experiencias del propio paciente oncológico (PO) como de su pareja se vuelven fundamentales al evaluar su relación, por lo que los objetivos del presente estudio fueron el examinar la manera en la que la experiencia de eventos hirientes y la calidez (reportada por el miembro de la pareja sano) afecta la satisfacción con su relación del PO, y explorar las diferencias y similitudes en la experiencia de EH de los PO, la mencionada calidez y la satisfacción con la relación de los PO a partir del tiempo de la misma y de cohabitar con la pareja, el estado civil y el número de hijos. Entre los principales resultados se halló que la presencia de EH se relacionó negativamente con la satisfacción marital, mientras que la calidez lo hizo de manera positiva, y algunas diferencias que evidencian que hay un mayor toque afectivo en las parejas con un menor tiempo de relación. Estos resultados se discuten a la luz de la teoría, mostrando evidencia sobre dichas variables en el contexto relacional.
\end{abstract}

Palabras clave: Cáncer; Eventos Hirientes; Calidez; Satisfacción marital; Pareja.

\begin{abstract}
Cancer affects the quality of life and health of those who suffer from it as well as their partners. Since the couple is a significant bond for people, the experience of hurtful events (HE) or warmth during daily interaction can cause satisfaction and dissatisfaction among its members. Thus, both the perceptions and experiences of cancer patients $(C P)$ and their partners become fundamental in the evaluation of their relationships, so the objectives of this research were: 1) to examine the way in which the experience of $H E$ in the $C P$ and warmth (given by the healthy partner) affects $C P$ satisfaction with their relationship and 2) explore differences and similarities in the experience of $H E$ from CPS, warmth (given by the healthy partner) and satisfaction with the relationship of the CPS based on the time in it, the time of cohabitation with the partner, the marital status and the number of children. Among the main results, it was found that the presence of HE was negatively related to marital satisfaction, while warmth was positively related; and some differences that show there is a
\end{abstract}

\footnotetext{
${ }^{1}$ Facultad de Psicología, Av. Universidad 3004, Col. Copilco Universidad, Coyoacán, 04510 Ciudad de México, México.
} 
greater affective touch in couples with a shorter relationship time. These results are discussed in the light of theory, showing evidence about said variables in the relational context.

Key words: Cancer; Hurtful events; Warmth; Marital satisfaction; Couple.

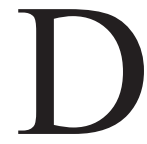
esde el siglo pasado se ha considerado el cáncer como un problema importante de salud pública en todo el mundo al ocasionar la pérdida de vidas debida a las deficiencias en las estrategias de prevención, diagnóstico y tratamiento de dicha enfermedad, además de cargas económicas, sociales y emocionales de gran magnitud. En México, esta enfermedad ocupa el tercer lugar en mortalidad, la que ha ido aumentando a lo largo de las últimas décadas (Rizo, González, Sánchez y Murguía, 2015).

A pesar de que algunas personas muestran cierta predisposición a la enfermedad, el comportamiento y el estilo de vida son factores que contribuyen en una mayor medida a su aparición (Gaviria, Vinaccia, Riveros y Quiceno, 2007). Por lo tanto, aspectos vinculados con la cotidianidad, como la familia, el trabajo, la búsqueda de bienestar o satisfacción personal, los logros y el buen establecimiento de relaciones personales resultan relevantes (Sprung, Janotha y Steckel, 2011). De hecho, durante la trayectoria de la enfermedad el paciente y las personas más cercanas pueden presentar modificaciones en su comportamiento y buscar cubrir las demandas del padecimiento, las que pueden variar según diversos elementos, como el grado y el tipo de incapacidad, el pronóstico de vida, el curso de la enfermedad, los protocolos de tratamiento y los síntomas experimentados (Mazzocco, Masiero, Carriero y Pravettoni, 2019). Más específicamente, la pareja proporciona la ayuda y un apoyo emocional tangible al paciente, lo que puede afectar la satisfacción con la relación (Mazzocco et al., 2019; Sprung et al., 2011). Aunado a ello, se ha encontrado que la pareja logra una adaptación más exitosa cuando sus miembros modifican su "intimidad" para incluir y trabajar las dificultades propias de la enfermedad, en vez de evadir posibles situaciones resultantes por las que se atraviesa a partir del padecimiento (Mazzocco et al., 2019). En este sentido, la enfermedad y las diversas variables propias de la pareja pueden influir en la satisfacción y el mantenimiento de la relación (Armenta, Sánchez-Aragón y Díaz-Loving, 2012; Sprung et al., 2011), lo que a su vez puede afectar de manera considerable la salud física y mental de ambos miembros (Mazzocco et al., 2019).

Así, la satisfacción en la pareja puede definirse como la evaluación que hace un individuo de 1) los sentimientos que le provocan diversos aspectos de su relación, 2) la interacción de los miembros y el tiempo de la relación, y 3) diversos elementos de la relación y la pareja (Armenta et al., 2012).

Cabe mencionar que hay otros aspectos que pueden afectar la satisfacción marital de manera negativa o positiva al resignificar la vida de la pareja en la cotidianidad y marcar las evaluaciones que se hacen del otro miembro y del propio vínculo (Armenta et al., 2012; Granillo y Sánchez-Aragón, 2020). Por un lado, están los eventos hirientes (EH en lo sucesivo), que se relacionan negativamente con la satisfacción (Granillo y Sánchez-Aragón, 2020) y que han sido identificados como transgresiones, devaluaciones relacionales o actos desfavorables que rompen ciertos acuerdos entre la pareja, como humillación, celos, indiferencia, amenazas, acusaciones, rechazo, infidelidad, evitación o violencia verbal o física, lo que trae consigo emociones negativas para la víctima, que se siente herida o lastimada profundamente por su pareja (Leary y Leder, 2009; Vangelisti, 2009).

No obstante, es importante señalar que el impacto con el que es percibido un EH depende de la manera en que la víctima lo conceptualiza, la familiaridad que siente respecto a ese tipo de situaciones y la manera en la que responde ante ello. De esta manera, habrá personas que pueden considerar como fugaz la causa de su dolor por la ocurrencia de un EH, otras que pueden considerarlo como algo grave e irrevocable, y otras más que pueden estar en un punto medio reconociendo que su pareja tuvo la intención de hacerles daño, pero considerando a la vez que es algo que se puede resolver (Bachman y Guerrero, 2006; Feeney, 2004).

Sin embargo, así como los EH han estado relacionados negativamente con la satisfacción de la 
pareja (Granillo y Sánchez-Aragón, 2020; Vangelisti y Young, 2000), hay otras variables que pueden afectarla de manera positiva, como la calidez (Ducat y Zimmer-GembeckLi, 2010; Valentine, Li, Meltzer y Tsai, 2020).

La calidez se ha definido como las demostraciones de afecto físico, conductual y verbal y como el acercamiento hacia el otro a través de abrazos, besos y palabras amorosas, así como compartir pensamientos y sentimientos mutuamente (Ducat y Zimmer-GembeckLi, 2010; Zepeda y Sánchez-Aragón, 2019). Es a través de dichas demostraciones afectivas como se va desarrollando, manteniendo y enriqueciendo un vínculo emocional característico, no parecido a ningún otro, conformado por la intimidad y el compromiso entre dos personas (Dibble, Levine y Park, 2012; Sánchez-Aragón, 1995).

Los aspectos que conforman la calidez (contacto físico, afectividad y cercanía) son elementos que han mostrado una asociación positiva con la satisfacción con la relación (Gulledge, Gulledge y Stahmannn, 2003; Triscoli, Croy, Olausson y Sailer, 2017) ya que dicha variable es fundamental para toda relación sana, pues favorece el conocimiento y la aceptación, fortalece el vínculo mediante el apoyo y la confianza, y representa el medio idóneo de reconocimiento mutuo, lo que hace posible el cumplimiento de las necesidades emocionales de los miembros de la pareja (Morales, 2016; Zepeda y Sánchez-Aragón, 2019).

Al tratarse de variables propias de las relaciones resultantes de la interacción entre dos personas cuyas particularidades matizan su dinámica, se ha visto que pueden ocurrir variaciones en función del tiempo y, en consecuencia, de la etapa en la que se encuentra la relación (Maureira, 2011; Pérez, Estrada, Pacheco, García y Tuz, 2017; Sánchez-Aragón, 2018). De esta manera, la diada se ve afectada por procesos evolutivos compuestos por el estatus de la relación, el tiempo de vida juntos, e incluso la presencia o ausencia de hijos o el número de estos, todo lo cual permea las prioridades, las funciones y, con ello, la calidad de la relación, lo que provoca cambios en las oportunidades de convivencia, en las relaciones sexuales, en la intimidad y en la manera de expresar las emociones y sentimientos verbal y físicamente (Sánchez-Aragón, 2018). Igualmente, diversos autores (Maureira, 2011; Sánchez-Aragón, 1995; 2018) han hecho propuestas referentes al ciclo vital de la pareja en cuanto al números de etapas y a las diferencias en el contenido de cada una. En este caso, se toma en consideración la propuesta de Sánchez-Aragón (2018) que considera cuatro etapas: desarrollo, mantenimiento con hijos pequeños, mantenimiento con hijos mayores y disolución o divorcio, siendo esta última una etapa que no necesariamente llegará.

Con base en lo anterior, el presente estudio correlacional (Hernández, Fernández y Baptista, 2014) explora el grado de asociación de la experiencia de EH y la calidez con la satisfacción relacional. Además, es de tipo comparativo (Cozby, 2005), en cuanto que se examina la manera en la que la experiencia de EH y la calidez reportada por el miembro sano de la pareja afecta la satisfacción con su relación del paciente oncológico (PO en lo sucesivo), así como porque explora las diferencias y similitudes en la experiencia de EH de los PO, la calidez reportada por el miembro sano de la pareja y la satisfacción con la relación de los PO a partir del tiempo en ella, el tiempo de cohabitar con la pareja, el estado civil y el número de hijos.

\section{MÉTODO}

\section{Participantes}

Se integró una muestra no probabilística por conveniencia (Hernández, Fernández y Baptista, 2010) de 194 parejas heterosexuales cuyos miembros debían cumplir con los siguientes criterios de inclusión: ser mayores de 18 años, ser uno de ellos $\mathrm{PO}$, tener una pareja romántica sana con la que se cohabitara, y haber alcanzado una escolaridad mínima de secundaria. En cuanto a los PO, participaron 141 mujeres y 53 hombres, heterosexuales, residentes de la Ciudad de México, cuyas edades oscilaron de 21 a 78 años $(M=47.98$, D.E. $=10.44)$. En términos de escolaridad, 48 contaban con estudios de secundaria, 57 con preparatoria y 89 con licenciatura. Respecto a su estado civil, 47 de ellos 
vivían en unión libre y 147 estaban casados, con un tiempo de relación de 1 año y 2 meses a 53.58 años $(\mathrm{M}=22.96$, D.E. $=11.59)$, y un tiempo de cohabitación de 8 meses a 53.58 años $(\mathrm{M}=21.30$, D.E. $=11.75)$. Del total de la muestra, 24 de los participantes indicaron no tener hijos, mientras que el resto reportó tener de uno a cinco. En relación son las parejas de los PO, participaron 194 personas (53 mujeres y 141 hombres), heterosexuales, residentes en la Ciudad de México, con edades de 18 a 77 años $(\mathrm{M}=49.13$, D.E. $=10.37)$, cuya escolaridad se distribuyó así: 42 indicaron estudios de secundaria, 66 de bachillerato y 86 de licenciatura.

\section{Instrumentos}

\section{Indicadores de Satisfacción con la Relación} (Sánchez-Aragón, 2013).

Incluye siete reactivos que se evalúan en una escala Likert de cinco opciones de respuesta (1, "Extremadamente mal", a 5, "Extremadamente bien") para indicar grado de satisfacción con diferentes aspectos de la relación ("Con mi relación de pareja me siento...", "Con respecto a la atención que recibo de mi pareja, me siento...", "Con el respeto que me brinda mi pareja me siento...", "Con la frecuencia con la que tenemos relaciones sexuales me siento...", "Con el cariño que recibo de mi pareja me siento...", "Con las actividades que comparto con mi pareja me siento..." y "Con la calidad de nuestras relaciones sexuales me siento...").

\section{Batería de Eventos Hirientes}

(Pérez y Sánchez-Aragón, 2015).

Este instrumento consta de una pregunta de selección múltiple, relativa a haber experimentado alguna situación con su pareja en la que la persona se sintiera herida u ofendida, con las siguientes opciones de respuesta: celos, crítica, indiferencia, infidelidad, decir que ya no le quiere, violencia verbal o física u otra situación u otra

Con el fin de conocer más la muestra de parejas en torno a los EH experimentados, se efectuaron los análisis de frecuencias que se presentan en la Tabla 1.
Tabla 1. Frecuencia de EH reportados por los participantes.

\begin{tabular}{|l|c|}
\hline \multicolumn{1}{|c|}{ Respuestas } & \multirow{2}{*}{ Frec. } \\
\cline { 1 - 2 } $\begin{array}{l}\text { He experimentado alguna situación con mi } \\
\text { pareja donde me he sentido herido(a) } \\
\text { y ofendido(a), como: }\end{array}$ & 73 \\
\hline $\begin{array}{l}\text { Celos (coquetear con otras personas, establecer } \\
\text { contactos con exparejas, etc.). }\end{array}$ & 36 \\
\hline $\begin{array}{l}\text { Crítica (burlas, insultarme de modo encubierto } \\
\text { o no, hacer comparaciones malintencionadas, } \\
\text { humillarme públicamente). }\end{array}$ & 69 \\
\hline $\begin{array}{l}\text { Indiferencia (preferir a otras personas } \\
\text { o situaciones). }\end{array}$ & 31 \\
\hline Infidelidad & 20 \\
\hline Decirme que ya no me quiere, alejarse de mí & 36 \\
\hline Ejercer violencia verbal o física & 15 \\
\hline Otro & \\
\hline
\end{tabular}

Dicha batería integra las siguientes tres subescalas, cuyos reactivos se evalúan en una escala Likert de cinco opciones de respuesta que indican grados de acuerdo:

1) Reacciones emocionales, con siete reactivos, que agrupan cuatro indicadores que explican en conjunto $62.79 \%$ de la varianza total, con un coeficiente alfa de Cronbach $(\alpha)$ de 0.83 , denominados: a) Daño (“¿Cuán herido se sintió?”), b) Rechazo ("¿Cuán rechazado por su pareja se sintió?”, “¿Cuán repudiado por su pareja se sintió?”), c) Control (“¿Cuánto control sintió que tuvo sobre la situación, en comparación con su pareja?”, “Cuánto poder le faltó en la situación, en comparación con su pareja?"), y d) Intencionalidad ("¿Usted cree que su pareja tenía la intención de herirlo/lastimarlo?”, “Usted cree que su pareja sabía que lo había herido/lastimado?");

2) Autopercepciones, con diez reactivos, agrupadas en dos factores que explican $52.9 \%$ de la varianza: a) Positivas (con adjetivos como "atractivo", "inteligente"...) $(\alpha=0.91)$ y $b)$ Negativas (con atributos como "tonto", "incompetente"...) $(\alpha=0.92), y$

3) Efectos de los EH, con ocho reactivos, agrupados en dos factores que explican 57.29\% de la varianza total: a) Efectos sobre sí mismo ("Me hizo menos seguro en situaciones similares 
en las que mis sentimientos fueron heridos"), con $\alpha=0.89$, y b) Efectos sobre la relación ("Esto hizo que la relación con mi pareja se debilitara temporalmente"), con $\alpha=0.87$.

Para medir la calidez se utilizaron las siguientes escalas:

\section{Escala de Toque Afectivo}

(Debrot, Schoebi, Perrez y Horn, 2013).

Conformada por seis reactivos en formato tipo Likert con cinco opciones de respuesta (de "Nunca o casi nunca" a "Más de una vez al día") que indican la frecuencia con la que se realiza alguna interacción física con la pareja, como tomarla de la mano, acostarse junto a ella, darle un masaje, abrazarla o besarla.

\section{Inventario de Afectividad en la Pareja}

(Sánchez-Aragón, 2018)

Este inventario está compuesto por 42 reactivos con formato de respuesta tipo Likert de cinco puntos (de "Nunca hago esto" a "Siempre hago esto"), distribuidos en tres factores que explican $68.75 \%$ de la varianza: 1) Apoyo $(\alpha=0.96)$, con 14 reactivos, como "Ayudarle con sus problemas" y "Apoyarle cuando lo necesita"; 2) Expresión de amor $(\alpha=0.96)$, con 16 reactivos, como "Tomarle la mano" y "Decirle «te amo»", y 3) Interacción y Comunicación $(\alpha=0.94)$, con 12 reactivos, como "Decirle que cuidaré de él/ella" y "Tocarle cada vez que puedo".

\section{Escala de Inclusión del Otro en el Yo}

(Aron, Aron y Smollan, 1992).

Validada en México por Sánchez-Aragón (1995), consta de un solo reactivo pictórico con respuesta tipo Likert de siete diagramas para indicar el grado de traslape de dos círculos como forma de definir la cercanía percibida en la relación de pareja.

\section{Procedimiento}

Los instrumentos fueron aplicados por cuatro psicólogas entrenadas en lugares donde fuera posible encontrar a los participantes, como clínicas, hospitales o albergues. En cada sitio se solicitaba verbalmente la participación voluntaria, confiden- cial y anónima de personas que cumplieran con los criterios de inclusión y que desearan participar respondiendo la batería de instrumentos. Se resolvieron las dudas en el momento y los resultados fueron puestos a su disposición en caso de que así lo desearan.

\section{Consideraciones éticas}

De acuerdo con el artículo 17 del Reglamento de la Ley General de Salud en Materia de Investigación para la Salud, el presente estudio se consideró de riesgo mínimo debido a que únicamente trata de la aplicación de pruebas psicológicas, para lo cual no se lleva a cabo ninguna manipulación de la conducta de los participantes, a quienes se plantean solamente las preguntas necesarias y se evita indagar en aspectos personales o de cualquier otra índole que nada aporten estadística y científicamente. Además, los participantes firmaron una carta de consentimiento informado verbal en la que se les informó que sus datos serían utilizados con fines científicos y que podían abandonar el estudio en cualquier momento sin consecuencia alguna. Todos los procedimientos se llevaron a cabo considerando el Código Ético del Psicólogo (Sociedad Mexicana de Psicología, 2010).

\section{Análisis de datos}

En cuanto al procedimiento para el análisis de datos, se utilizó el programa estadístico SPSS Statistics. v. 21, mediante el cual se realizó una prueba de normalidad (Kolmogorov-Smirnov) para comprobar el cumplimiento del supuesto de normalidad de las variables estudiadas en la muestra. Dado que dicho principio se cumplió, se utilizó estadística paramétrica, aceptándose la hipótesis nula que indica homogeneidad en las muestras.

A partir de ello, y en busca de cubrir el primer objetivo. se llevó a cabo una correlación producto-momento de Pearson para examinar la relación entre las variables, y después análisis de varianza de una vía con el fin de cumplir el segundo objetivo, orientado a explorar las diferencias entre las variables según el tiempo de relación y de cohabitación, el estado civil y el número de hijos. 


\section{RESULTADOS}

\section{EH, calidez y satisfacción marital}

Se efectuó una correlación producto-momento de Pearson con el fin de examinar la asociación de la experiencia de EH de los PO, la calidez indicada por sus parejas y la satisfacción con la relación.
Los resultados, que se muestran en la Tabla 2, indican que cuando los PO perciben que su pareja tuvo una mayor intención de hacerles daño a través del $\mathrm{EH}$, obtuvieron menos satisfacción con el cariño recibido, las actividades compartidas con la pareja, la relación en general, la calidad de las relaciones sexuales y el respeto brindado por el otro.

Tabla 2. Correlación de la experiencia de EH con la satisfacción con la relación en el PO.

\begin{tabular}{|c|c|c|c|c|c|c|c|c|}
\hline & \multicolumn{7}{|c|}{ Satisfacción con... } \\
\hline & & 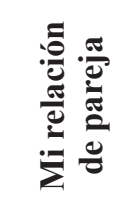 & 预 & 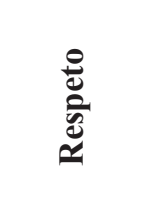 & 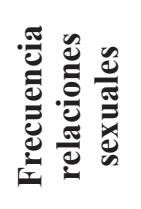 & Uֶ: & 莺 & 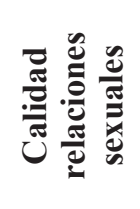 \\
\hline \multirow{3}{*}{ Reacciones } & Rechazo & & & & & & & \\
\hline & Control & & & & & & & \\
\hline & Intención & $-.292 * *$ & $-.272 * *$ & $-.266 * *$ & & $-.309 * *$ & $-.294 * *$ & $-.280 * *$ \\
\hline \multirow{2}{*}{ Autopercepción } & Positiva & & & & & & & \\
\hline & Negativa & $-.227 * *$ & & & $-.228 * *$ & $-.223 * *$ & $-.245 * *$ & $-.316 * *$ \\
\hline \multirow{2}{*}{ Efectos } & En sí mismo & $-.223 * *$ & & $-.217 * *$ & & $-.203 * *$ & $-.229 * *$ & $-.206^{* *}$ \\
\hline & En la relación & $-.265^{* *}$ & $-.309^{* *}$ & $-.267 * *$ & & $-.255^{* *}$ & $-.268 * *$ & \\
\hline
\end{tabular}

** Correlación significativa al nivel de 0.01 (bilateral)

De manera similar, se observó que cuando el EH propiciaba una mayor autopercepción negativa en el PO, se favorecía una menor satisfacción respecto a la calidad de las relaciones sexuales y las actividades compartidas en pareja, seguida por la frecuencia con la que se tenían relaciones sexuales, con la relación en general y con el cariño recibido por la pareja. En la medida en que los PO experimentaban más efectos negativos en sí mismos, ocasionando con ello que sintieran decaer su autoestima, más inseguridad y vulnerabilidad de ser lastimados posteriormente), experimentaban menor satisfacción en las actividades realizadas en pareja, en la relación en general, en el respeto brindado por el otro, en la calidad de las relaciones sexuales y en el cariño ofrecido por la pareja.

Por último, los hallazgos indicaron que cuando los PO percibían más daño en su relación por el EH -es decir, un debilitamiento temporal o definitivo y más sentimientos de desconfianza hacia la pareja- mostraban una menor satisfacción con la atención recibida del otro, con las actividades conjuntas, con el respeto brindado por la pareja, con la relación y al cariño recibido (ver Tabla 2).

Por otra parte, respecto a la asociación entre la calidez proveniente de la pareja (contacto afectivo, afectividad y cercanía) y la satisfacción que el PO tiene con su relación, los datos indicn que a mayor contacto afectivo (besar, abrazar y tocar zonas erógenas del cuerpo), mayor apoyo brindado en momentos de necesidad, mayor expresión de amor y mejor interacción y comunicación, esto es, que la satisfacción con todas las áreas exploradas se ve beneficiada. Particularmente, se observó que la satisfacción con la relación, con la atención, el respeto y cariño del PO se beneficiaba principalmente del apoyo que su pareja le daba; la satisfacción con la frecuencia y calidad de las relaciones sexuales del paciente fue enriquecida básicamente por la cercanía y la satisfacción con las actividades compartidas, favorecidas por la interacción y la comunicación entre el paciente y su pareja (Tabla 3). 
Tabla 3. Correlación de la calidez proveniente de la pareja y la satisfacción con la relación en el PO.

\begin{tabular}{|c|c|c|c|c|c|c|c|c|}
\hline & \multicolumn{7}{|c|}{ Satisfacción con... } \\
\hline & & 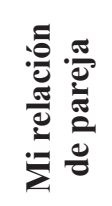 & & 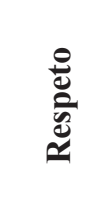 & 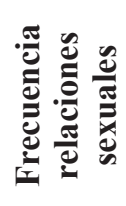 & שֶ: & 窇 & 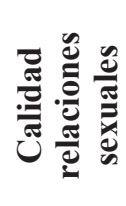 \\
\hline \multirow{5}{*}{ 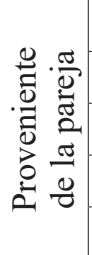 } & Contacto afectivo & $.296 * *$ & $.315^{* *}$ & $.229 * *$ & $.280 * *$ & $.242 * *$ & $.233 * *$ & $.317 * *$ \\
\hline & Apoyo & $.310 * *$ & $.317 * *$ & $.248 * *$ & $.230 * *$ & $.267 * *$ & $.236 * *$ & $.242 * *$ \\
\hline & Expresión de amor & $.236 * *$ & $.273^{* *}$ & & $.234 * *$ & $.208^{* *}$ & $.220 * *$ & $.259 * *$ \\
\hline & Interacción y comunicación & $.298 * *$ & $.259^{* *}$ & $.202 * *$ & $.254 * *$ & $.216^{* *}$ & $.267 * *$ & $.315^{* *}$ \\
\hline & Cercanía & $.237 * *$ & $.203 * *$ & & $.399 * *$ & $.194 * *$ & $.237 * *$ & $.401 * *$ \\
\hline
\end{tabular}

** Correlación significativa al nivel de 0.01 (bilateral).

\section{Tiempo de la relación}

Posteriormente y con el fin de satisfacer el segundo objetivo, orientado a explorar las diferencias en la experiencia de EH, la calidez por parte de la pareja y la satisfacción con la relación en los PO tomando como variable de clasificación el tiempo de la misma, se realizaron cortes equivalentes a la muestra considerando el 33\% del total de los participantes. Así, quedaron tres grupos: 1) de 14 meses a 18.41 años, 2) de 18.75 a 26.91 años, y 3) de 27 a 53.58 años, mismos que se utilizaron para realizar un análisis de varianza de una vía.

Los resultados indican semejanza en los grupos respecto a la experiencia de EH, la calidez -excepto para el instrumento de toque afectivo-y la satisfacción marital. Tales diferencias estadísticamente significativas mostraron un mayor toque afectivo brindado por las parejas de los PO del grupo 1 con un tiempo de relación más corto $(\mathrm{M}=3.39$, D.E. $=1.02, \mathrm{n}=61)$, en comparación con el grupo 3, con una relación de más tiempo $(\mathrm{M}=2.70$, D.E. $=1.39, \mathrm{n}=66)$, en cuanto a la frecuencia con la que indicaron tomar de la mano a la pareja, abrazarla, besarla, darle masajes y tener sexo, siendo de $\mathrm{F}(2[15.33]=4.98, p=0.00)$ el tamaño del efecto.

\section{Tiempo de vivir juntos}

Además, se llevó a cabo un análisis de varianza de una vía utilizándose grupos realizados a partir de cortes equivalentes a la muestra, considerando $33 \%$ según el tiempo que tenían las parejas de vi- vir juntas. Se obtuvieron tres grupos: 1) de 8 meses a 16.16 años, 2) de 16.66 a 25.25 años, y 3) de 25.50 a 53.58 años. A partir de ello, los resultados fueron similares a los del tiempo en la relación indicados anteriormente; es decir, se observó similitud respecto a la experiencia de $\mathrm{EH}$, la calidez (excepto para el instrumento de toque afectivo y el factor de expresión de amor) y la satisfacción marital. Las diferencias estadísticamente significativas obtenidas para la calidez en la parte de toque afectivo señalaron que los participantes del grupo 1, con un tiempo de vivir juntos más corto $(\mathrm{M}=3.33$, D.E. $=1.16, \mathrm{n}=63)$ indicaron tocar a su pareja en mayor medida, tomándola de la mano, abrazándola, dándole masajes y teniendo relaciones sexuales con ella, en comparación con el grupo 3, con un tiempo de cohabitación mayor $(\mathrm{M}=2.54$, D.E. $=1.40, \mathrm{n}=64)$ y con un tamaño del efecto de $\mathrm{F}(2,21.97)=7.31, p=0.00$, quienes indicaron cohabitar con su pareja desde hacía más tiempo.

Del mismo modo, se observaron diferencias estadísticamente significativas respecto al factor de expresión de amor, perteneciente a la variable de afectividad, que indican que el grupo $2(\mathrm{M}=4.12$, D.E. $=0.89, \mathrm{n}=64)$ refirió realizar en mayor medida acciones con su pareja, tales como tomarla de la mano y decirles "Te amo", en comparación con el grupo $3(\mathrm{M}=3.62$, D.E. $=1.16, \mathrm{n}=64)$, con un tamaño del efecto de $\mathrm{F}(2,9.42)=4.40, p=0.01$, quienes llevaban más tiempo viviendo con su pareja. 


\section{Estado civil}

En cuanto a la búsqueda de diferencias por estado civil, se efectuó una prueba $t$ de Student para muestras independientes, en la cual no se encontraron diferencias significativas al nivel de $p<.05$, pero sí similitudes en todas las variables estudiadas.

\section{Número de hijos}

Finalmente, se efectuó un análisis de varianza de una vía con el fin de examinar las diferencias en la experiencia de EH por el $\mathrm{PO}$, la calidez por parte de la pareja de los PO y la satisfacción con la relación en estos mismos participantes, en función del número de hijos reportado. Con base en ello, se conformaron tres grupos: 1) participantes sin hijos, 2) participantes con uno o dos hijos y 3) participantes con tres o más hijos. Los hallazgos muestran similitud en cuanto a la experiencia de $\mathrm{EH}$, la calidez - excepto para el factor de la expresión de amor- y la satisfacción marital. Así, las diferencias estadísticamente significativas para la expresión de amor, con un tamaño del efecto de $\mathrm{F}(2,8.80)=3.91, p=0.02)$, muestran que las parejas de los PO del grupo $2(\mathrm{M}=4.05$, D.E. $=1.02$, $\mathrm{n}=73$ ) indicaron expresarles amor con acciones tales como tomarlos de la mano y decirles " $\mathrm{Te}$ amo" en mayor medida que las parejas de los PO del grupo $3(\mathrm{M}=3.55$, D.E. $=1.19, \mathrm{n}=52)$.

\section{DISCUSIÓN}

La presente investigación se orientó, en primer lugar, a examinar la relación de la experiencia de $\mathrm{EH}$ de los PO y la calidez proveniente de sus parejas en su satisfacción con la relación. Previamente a los análisis de correlación, se registraron las frecuencias de los principales EH experimentados por las parejas participantes de esta investigación, encontrándose que lo que más habían vivido los PO eran celos, indiferencia, crítica, violencia e infidelidad, seguidos por el alejamiento y otras razones, lo que implica que en estas parejas los EH mencionados se unen a la experiencia del cáncer, con sus posibles consecuencias emocionales en el estado de salud del PO (Badr, 2017; Mazzocco et al., 2019;
Sprung et al., 2011). En este mismo tópico, y tras los análisis pertinentes, se observó que una mayor intención percibida de herir o hacer daño ante un EH se relacionó con una menor satisfacción con el cariño recibido y con las actividades compartidas con la pareja, así como con una menor satisfacción con la relación en general, con la calidad de las relaciones sexuales y con el respeto brindado por el otro. Respecto a esto, Vangelisti y Young (2000) señalan que quienes valoran como intencional un comportamiento negativo del otro pueden tener una visión diferente del impacto de dicho EH en la relación con tal persona. Aquí, los individuos evalúan dicha intencionalidad a partir de diversos elementos (voluntad a realizar el comportamiento, previsión o planificación y conocimiento de las consecuencias) para considerar como fugaz o como grave e irrevocable la causa de su dolor (Bachman y Guerrero, 2006; Feeney, 2004). De esta manera, la forma en que las personas perciben el comportamiento del otro en las relaciones de pareja está asociado con sus sentimientos dentro de la relación, de modo que quienes interpretan dicha acción de una manera que favorece la relación tienden a estar más satisfechas que aquellos que se centran en los aspectos negativos, manteniendo sentimientos de angustia ante el pensamiento de posible intencionalidad, provocando insatisfacción en la relación, y sintiéndose además menos cerca de su pareja (Feeney, 2004; Granillo y Sánchez-Aragón, 2020; Vangelisti y Young, 2000). Debe decirse que todo lo anterior puede verse magnificado debido a la presencia del padecimiento, el cual se ha encontrado íntimamente relacionado con las emociones del PO y en sus relaciones interpersonales, principalmente con la pareja (Badr, 2017; Sprung et al., 2011). De igual manera, la satisfacción y los EH pueden funcionar de manera bidireccional en virtud de que es poco probable que las personas que se encuentren muy satisfechas con su relación busquen distanciarse de la misma después de un evento negativo, no obstante haberlo percibido como hiriente (Vangelisti y Young, 2000).

En congruencia con lo anterior, se halló que una mayor autopercepción negativa de los PO al haber experimentado EH dentro de su relación se asoció con una menor satisfacción respecto a la 
calidad de las relaciones sexuales y las actividades compartidas en pareja, seguido por una menor satisfacción con la frecuencia con la que mantienen relaciones sexuales, con la relación en general y con el cariño recibido de la pareja. Respecto a esto, Granillo, Sánchez-Aragón y Gómez (2020), al igual que Vangelisti (2009), citan hallazgos en los que se observa que las transgresiones tienden a causar daño físico o psicológico en diversos niveles dentro de una relación de pareja; al ser consideradas como una lesión personal, pueden provocar un efecto desfavorable a la autoestima debido a que amenazan los modelos mentales positivos propios del individuo, resultando en que la persona -en este caso el PO- se sienta insuficiente respecto a su capacidad para lograr cubrir sus propias necesidades y las del otro miembro de la relación. Esto último es algo de suma importancia en la satisfacción marital (Granillo y Sánchez-Aragón, 2020; Romero, Galicia y Del Castillo, 2017) teniendo en cuenta que la capacidad para destruir ese sentido de seguridad por lo sucedido está relacionado con las opiniones que se tengan sobre uno mismo con anterioridad y con el tipo de relación que se tenga con el otro (Shaver, Mikulincer, Lavy y Cassidy, 2009; Vangelisti, 2009), sumado a que el PO y su pareja atraviesan por una situación que por sí misma ya conlleva diversas implicaciones físicas, psicológicas y sociales, lo que puede contraponerse a un adecuado ajuste emocional (Sprung et al., 2011).

Además, cuando los PO perciben inseguridad, un decaimiento de su autoestima, vulnerabilidad a ser lastimados nuevamente, percepción de que se afecta la relación, provocando con ello su debilitamiento temporal o definitivo, así como sentimientos de desconfianza hacia la pareja después de haber atravesado por un EH, muestran menos satisfacción con la atención recibida, las actividades realizadas en pareja, la relación en general, el respeto brindado por el otro, seguidos por una menor satisfacción respecto a la calidad de las relaciones sexuales y el cariño ofrecido por la pareja. Concerniente a esto, autores como Mazzoco et al. (2019) y Rizo et al. (2015) señalan que la autoestima de los PO puede verse deteriorada como consecuencia de los tratamientos y sus efectos secundarios, los posibles cambios físicos y la incertidumbre ante la evolución de la enfermedad, maximizando además los sentimientos ante diversas experiencias de la vida diaria y, por supuesto, las relacionadas con la interacción de pareja. Además, se ha comprobado el impacto personal asociado a la ocurrencia de un EH como consecuencia del daño al sentido de seguridad de una persona, así como a la manera de pensar u opinar sobre sí mismo y los demás, lo que resulta en la expresión de sentimientos negativos, como dolor, ira, tristeza y angustia (Leary y Leder, 2009; Vangelisti, 2009). por solo mencionar algunos. De esta manera, los sentimientos negativos desestabilizan la relación (Romero et al., 2017) y afectan la satisfacción que los PO sienten respecto a lo que reciben y comparten con su pareja (Lavner y Bradbury, 2012).

Por otra parte, respecto a la relación de la calidez (contacto afectivo, afectividad y cercanía) provista por la pareja para la satisfacción del PO con su vínculo, se observó que un mayor contacto afectivo mediante besos, abrazos y tocamientos de zonas las erógenas del cuerpo se traducen en un mayor apoyo brindado en momentos de necesidad y una mayor expresión de amor con acciones como tomarle la mano a la pareja y decirle que se le ama, así como una mayor interacción y comunicación indicados por las parejas de los pacientes se relaciona con una mayor satisfacción en todas las áreas examinadas en el presente estudio. Dichos hallazgos se ven apoyados por Armenta et al. (2012) y Otero y Flores (2016), quienes indican que la satisfacción marital puede apreciarse a través de un modelo multidimensional que incluye elementos intercambiados mediante la convivencia que permiten el conocimiento de la pareja, la intimidad y el acercamiento, y contribuyen a que sus miembros se perciban con cercanía y manifiesten su afecto física y verbalmente, lo que resulta positivo en la satisfacción con la relación. Asimismo, el tiempo compartido, el conocimiento mutuo, las muestras de apoyo, confianza y afecto, son aspectos considerados por las personas para indicar una conexión especial entre los miembros de la pareja (Dibble et al., 2012; Granillo y Sánchez-Aragón, 2021) y que, sumados a diversas manifestaciones de amor que resulten en que el PO se sienta querido y valorado por el otro, favorecen 
una relación sana de aceptación y vínculos fortalecidos que van de la mano con una satisfacción marital positiva (Morales, 2016; Zepeda y Sánchez-Aragón, 2019).

Aunado a lo anterior, se notó particularmente que el apoyo brindado en momentos importantes por las parejas de los PO favorece la satisfacción de estos últimos con la atención, el respeto y el cariño brindado por sus parejas, mientras que una mayor interacción y comunicación entre el PO y su pareja se relaciona con una mayor satisfacción en el PO en cuanto a las actividades compartidas. Aunado a esto, Morales (2016) y Guerrero, Andersen y Afifi (2018) indican que el afecto presente en el sustento, la ayuda y la protección al PO por parte de su pareja refleja también aceptación y produce bienestar, expresión de amor e inmediatez ( $c f$. Perales, Jiménez y Sánchez-Aragón, 2014). En dichos elementos se encuentra inmerso también el respeto interpersonal, lo que contribuye a la armonización de los derechos propios y los de la otra persona, indispensable para una relación funcional y satisfactoria (Capafóns y Sosa, 2015). Resulta así que la afectividad física y verbal, el apoyo y la cercanía favorecen la calidez en la relación y, con ello, la aceptación y el respeto de la pareja que, finalmente, se traduce en satisfacción (Capafóns y Sosa, 2015; Morales, 2016; Zepeda y Sánchez-Aragón, 2019).

La cercanía en la relación percibida por los participantes se vincula principalmente con la satisfacción respecto a la frecuencia y calidad de las relaciones sexuales, lo que es congruente con la visión de que la cercanía proporciona una interconexión particular entre los miembros de la relación, punto este que se ve apoyado por Ortega (2012) y Pérez et al. (2017), quienes encontraron que variables relacionales y de afecto, como las demostraciones de cariño y afectividad en el grado deseado entre la pareja son predictores importantes de la satisfacción sexual positiva, un componente básico de la sexualidad en la pareja que favorece la interacción.

En lo que respecta al segundo objetivo, encaminado a examinar las diferencias en la experiencia de $\mathrm{EH}$, la calidez brindada por parte de las parejas de los PO y la satisfacción marital en estos últimos a partir del tiempo de relación, el tiempo de vivir juntos, el estado civil y el número de hijos, se obtuvieron algunos resultados interesantes que se discuten a continuación.

En cuanto a la experiencia de EH, se observó que los pacientes indican de forma semejante la percepción de rechazo por parte de la pareja, los sentimientos de control ante la situación, la intencionalidad, los cambios en su autopercepción positiva o negativa, las afectaciones personales (decaimiento de la autoestima, inseguridad y vulnerabilidad) y relacionales (desconfianza del otro, debilitamiento temporal o definitivo de la relación), sin importar el tiempo de relación y de vivir con la pareja, el estado civil ni el número de hijos. Ello se explica porque los EH son situaciones negativas que pueden ocurrir en cualquier momento y en diferentes relaciones interpersonales (pareja, amigos, hermanos, etc.); sin embargo, son eventos más vinculados al tipo de relación y a la cercanía percibida por el otro (Vangelisti, 2009), así como a la percepción de cada persona respecto a la magnitud del daño que pueden llegar a causar (Bachman y Guerrero, 2006; Feeney, 2004) y los comportamientos de los involucrados en la situación (Shaver et al., 2009; Vangelisti, 2009) y no tanto con el tiempo que tenga la relación. Si bien la personalidad y la forma de reacción o afrontamiento ante los conflictos son aspectos individuales que influyen en la interacción con los otros, no dependen de esta (Gaviria et al., 2007; Romero et al., 2017). De igual forma, los hallazgos en cuanto a los hijos pueden explicarse debido a que los EH involucran a los miembros de la pareja en cuanto a la propia percepción de cada uno, y que tienen que ver con variables de tipo individual, como la autoestima, la autopercepción, la confianza y la seguridad (Feeney, 2004; Vangelisti, 2009), por lo que los hijos pueden estar involucrados en algún conflicto debido a ciertos aspectos relacionados con la crianza (Estévez, 2013; Rivera-Aragón y Díaz-Loving, 2002). No obstante, el número de hijos puede no representar un cambio en dichas percepciones o maneras de ajuste psicológico ante un conflicto con la pareja y la satisfacción marital propiamente (Guzmán y Contreras, 2012).

En cuanto a la calidez (contacto afectivo, afectividad y cercanía), las parejas de los pacientes reportaron de manera semejante el apoyo brindado 
y la interacción y comunicación con sus parejas mediante detalles y halagos, sin importar el tiempo de relación y de cohabitación con su pareja, el estado civil ni el número de hijos. A este respecto, se ha hallado que las demostraciones de afecto en sus diversas formas son fundamentales en toda relación sana y duradera debido a que favorece el fortalecimiento de los vínculos $\mathrm{y}$, con ello, el reconocimiento mutuo dentro de la pareja (Morales, 2016; Zepeda y Sánchez-Aragón, 2019). Además, se ha encontrado que una vez establecida la relación, lo que adquiere importancia es lograr que permanezca y se prolongue lo más posible, lo que hace que las personas utilicen diversas estrategias, como las demostraciones de afecto y calidez a través del apoyo, los halagos y las expresiones de amor verbales y físicas, siendo necesarios tales elementos para ese propósito al lograr que el PO se sienta aceptado, querido y valorado por el otro, lo que fortalece el referido vínculo (Guerrero et al., 2018; Zepeda y Sánchez-Aragón, 2019).

Por último, en cuanto a la satisfacción marital, los resultados fueron parecidos, es decir, los PO, sin importar el tiempo de su relación de pareja, mostraron de igual manera sentirse satisfechos con la relación en general, la atención, el respeto y el cariño recibidos por la pareja, así como con las actividades compartidas y la calidad y frecuencia de las relaciones sexuales. A ese respecto, Armenta et al. (2014) y Sánchez-Aragón (2018) señalan ciertas características esperadas en cada momento durante el desarrollo de la relación; sin embargo, la percepción de dichos momentos no es la misma para ambos miembros debido a que cada uno interpreta y vive la relación de manera única según las experiencias y expectativas individuales. No obstante, cuando estas diferencias se ven minimizadas, las parejas se sienten más satisfechas con su relación (Armenta et al., 2014). Esto último puede explicar los resultados obtenidos al considerar que la mayoría de los PO indicaron tener relaciones de varios años; el haber alcanzado estadios más avanzados de la relación habían logrado cierta estabilidad, lo que contribuye a la minimizar los momentos antes descritos y, con ello, lograr satisfacción y un bienestar constante (Estévez, 2013; Nina, 2011).
Sin embargo, las diferencias significativas se encontraron en la calidez. Para el toque afectivo se observó que las parejas de los PO con un menor tiempo de relación mostraron tomar de la mano, besar y abrazar en mayor medida a los PO que quienes tenían una relación más duradera. Lo mismo ocurrió en cuanto al tiempo de cohabitación: en las parejas que llevaban viviendo juntas un tiempo intermedio (grupo 2) se observó un contacto afectivo más frecuente que en quienes llevaban más tiempo. Por último, referente a la expresión de amor, se pudo apreciar que las parejas de los participantes con un tiempo intermedio de vivir en pareja indicaron tener más expresiones amorosas que quienes tenían más tiempo. Asimismo, fueron las parejas con dos hijos las que indicaron tener más de tales expresiones que quienes tenían tres o más hijos. Ya Maureira (2011) y Sánchez-Aragón (2018) han señalado que el componente romántico de la relación conformado por conductas de intimidad y pasión puede disminuir significativamente con el paso del tiempo, la costumbre y la disminución de los deseos por conquistar al otro. Así, aunque se ha visto que la parte afectiva es un elemento crucial de la relación de pareja, es posible que algunos aspectos, como el afecto físico, disminuyan debido a los cambios en la vida de la pareja: el arribo de los hijos y las demandas que ello implica, lo que da paso a una renegociación del estilo de vida y de las pautas de interacción de los miembros de la relación (Armenta et al., 2012; Pérez et al., 2017). De esta manera, los presentes hallazgos concuerdan con lo descrito acerca de las etapas de la pareja de Sánchez-Aragón (2018), quien apunta que las parejas con hijos priorizan los aspectos relacionados con el desarrollo, el cuidado y la educación de los mismos, y buscan además equilibrar los tiempos destinados al trabajo, el hogar y, por supuesto, la pareja. Sin embargo, con la llegada de los hijos la pareja tiene menos tiempo para compartir y, por ende, experimenta una disminución o la falta de intimidad (Sánchez-Aragón, 1995, 2018). Aun cuando los hijos crezcan, la pareja sigue afrontando diversos retos relativos a su crianza y experimentando cambios y posibles pérdidas económicas, sociales y biológicas al tratar de adecuarse a las demandas de aquellos y per- 
cibiendo por ello una menor satisfacción marital (Armenta et al., 2012; Sánchez-Aragón, 2018). Con base en lo anterior, la llegada de los hijos es una etapa destacada en la relación de pareja, y puede ser más demandante conforme el número de hijos aumenta (Sánchez-Aragón, 1995).

En conclusión, los presentes resultados evidencian que la satisfacción con la relación se asocia con variables negativas (presencia de EH dentro de la pareja) y positivas (afectividad, toque afectivo y cercanía entre sus miembros). Además, la calidez favorece una mayor satisfacción con la relación en los distintos ámbitos que incluye, ocurriendo lo contrario cuando de un EH se trata.

Respecto a las limitaciones de este estudio, debe resaltarse que los datos aquí presentados muestran el análisis de la información obtenida considerando al cáncer como un padecimiento gene- ral, sin diferenciar los diversos tipos de la enfermedad y las posibles variaciones que puede haber sobre el tiempo de diagnóstico y el tratamiento. Además, otras características individuales de la muestra, como la edad de los participantes y el sexo, no fueron consideradas debido a que se buscó centrar el estudio en variables relacionales para el cumplimento de los objetivos planteados.

Por lo anterior, se propone continuar el estudio de las variables señaladas en personas con otros padecimientos debido a que las condiciones $y$ el contexto en el que se vive cuando se sufre una enfermedad pueden afectar otras variables individuales y relacionales presentes en el citado vínculo. Aunado a ello, se pretende continuar trabajando con los datos obtenidos a fin de elaborar materiales de apoyo para las parejas que conviven con el cáncer.

\section{AGRADECIMIENTOS}

Esta investigación fue realizada gracias al Programa UNAM-PAPITT IN304919 del proyecto intitulado "Factores protectores y de riesgo a la salud en parejas sanas y con enfermedad crónico-degenerativa".

Citación: Granillo-Velasco, L.F. y Sánchez-Aragón, R. (2021). Satisfacción marital en pacientes oncológicos: su relación con los eventos hirientes y la calidez brindada por su pareja. Psicología y Salud, Número Especial, 57-70. https://doi.org/10.25009/pys.v31i3.2728.

\section{REFERENCIAS}

Armenta H., C., Sánchez-Aragón, R. y Díaz-Loving, R. (2012). ¿De qué manera el contexto afecta la satisfacción con la pareja? Summa Psicológica, 19(2), 51-62.

Armenta H., C., Sánchez-Aragón, R. y Díaz-Loving, R. (2014). Efectos de la cultura sobre las estrategias de mantenimiento y satisfacción marital. Acta de Investigación Psicológica, 4(2), 1572-1584.

Aron, A., Aron, E.N. y Smollan, D. (1992). Inclusion of Other in the Self Scale and the structure of interpersonal closeness. Journal of Personality and Social Psychology, 63(4), 596-612. Doi: 10.1037/0022-3514.63.4.596.

Bachman, G. y Guerrero, L. (2006). Relational quality and communicative responses following hurtful events in dating relationships: An expectancy violations analysis. Journal of Social and Personal Relationships, 23(6), 943-963. Doi: $10.1177 / 0265407506070476$.

Badr, H. (2017). New frontiers in couple-based interventions in cancer care: refining the prescription for spousal communication. Acta Oncologica, 56(2), 139-145. Doi: 10.1080/0284186X.2016.1266079.

Capafóns J., I. y Sosa C., D. (2015). Relaciones de pareja y habilidades sociales: El respeto interpersonal. Behavioral Psychology/ Psicología Conductual, 23(1), 25-34.

Cozby, P. (2005). Methods in Behavioral Research. New York: McGraw-Hill.

Debrot, A., Schoebi, D., Perrez, M. y Horn, A.B. (2013). Touch as an interpersonal emotion regulation process in couples' daily lives: The mediating role of psychological intimacy. Personality and Social Psychology Bulletin, 39(10), 1373-1385. Doi: $10.1177 / 0146167213497592$. 
Dibble, J.L., Levine, T.R. y Park, H.S. (2012). The Unidimensional Relationship Closeness Scale (URCS): Reliability and validity evidence for a new measure of relationship closeness. Psychological Assessment, 24(3), 565-572. Doi: 10.1037/a0026265.

Ducat, W.H. y Zimmer-Gembeck, M.J. (2010). Romantic partner behaviors as social context: Measuring six dimensions of relationships. Journal of Relationships Research, 1(1), 1-16. Doi: 10.1375/jrr.1.1.1.

Estévez C, J.L. (2013). La pareja: un análisis cualitativo de su relación basada en apego y/o desapego, por etapas que integran el lado oscuro del ciclo de acercamiento-alejamiento de Díaz-Loving. Tesis inédita de doctorado. México: Universidad Iberoamericana. Recuperado de http://ri.ibero.mx/bitstream/handle/ibero/598/015792s.pdf?sequence $=1$.

Feeney, J.A. (2004). Hurt feelings in couple relationships: Towards integrative models of the negative effects of hurtful events. Journal of Social and Personal Relationships, 21(4), 487-508. Doi: 10.1177/0265407504044844.

Gaviria G., A.M., Vinaccia A., S., Riveros J., M.F. y Quiceno S., J.M. (2007). Calidad de vida relacionada con la salud, afrontamiento del estrés y emociones negativas en pacientes con cáncer en tratamiento quimioterapéutico. Psicología desde el Caribe, 20, 50-75.

Granillo V., L.F. y Sánchez-Aragón, R. (2020). Deteriorando la salud en pareja. Enseñanza e Investigación en Psicología, 2(2), 203-215. https://revistacneip.org/index.php/cneip/article/view/107.

Granillo V., L.F., Sánchez-Aragón, R. y Gómez B., G.A. (2020). Eventos hirientes, amor compasivo y calidad de vida en parejas con cardiopatía. Psicumex, 10(2), 45-70. Doi: 10.36793/psicumex.v10i2.350.

Granillo V., L.F. y Sánchez-Aragón, R. (2021). Altruism, uncontrollability and affectivity in physical health of partners of patients with diabetes. En R. Sánchez-Aragón (Ed.): Diabetes and couple relationships: Protective and risk factors (pp. 93-114). New York: Springer International Publishing.

Guerrero, L.K., Andersen, P.A. y Afifi, W.A. (2018). Close Encounters: communication in relationships. Townsend Oaks, CA: Sage Publications.

Gulledge, A.K., Gulledge, M.H. y Stahmannn, R.F. (2003). Romantic physical affection types and relationship satisfaction. The American Journal of Family Therapy, 31(4), 233-242. Doi: 10.1080/01926180390201936.

Guzmán G., M. y Contreras G., P. (2012). Estilos de apego en relaciones de pareja y su asociación con la satisfacción marital. Psykhe, 21(1), 69-82. Doi: 10.4067/S0718-22282012000100005.

Hernández S., R., Fernández C., C. y Baptista L., P. (2010). Metodología de la investigación. México: McGraw-Hill.

Lavner, J.A. y Bradbury, T.N. (2012). Why do even satisfied newlyweds eventually go on to divorce? Journal of Family Psychology, 26(1), 1-10. Doi: 10.1037/a0025966.

Leary, M. y Leder, S. (2009). The nature of hurt feelings: Emotional experience and cognitive appraisals. En A. Vangelisti (Ed.): Feeling hurt in close relationships (pp. 15-33). Cambridge (UK): Cambridge University Press.

Maureira C., F. (2011). Los cuatro componentes de la relación de pareja. Revista Electrónica de Psicología Iztacala, 14(1), 321-332.

Mazzocco, K., Masiero, M., Carriero, M.C. y Pravettoni, G. (2019). The role of emotions in cancer patients' decision-making. Ecancermedicalscience, 13, 1-22. Doi: 10.3332/ecancer.2019.914.

Morales T., D.M. (2016). Afecto y relación de pareja. Tesis inédita de licenciatura. Ciudad de Guatemala: Universidad Rafael Landívar.

Nina E., R. (2011). ¿Qué nos mantiene juntos? Explorando el compromiso y las estrategias de mantenimiento en la relación marital. Revista Intercontinental de Psicología y Educación, 13(2), 197-220.

Ortega R., J. (2012). El vínculo de pareja: Una posibilidad afectiva para crecer. Revista Electrónica Educare, 16(Núm. Esp.), 23-30. Doi: 10.15359/ree.16-Esp.3.

Otero R., M.D.P. y Flores G., M.M. (2016). Funcionamiento familiar como predictor de la satisfacción marital en hombres y mujeres. Enseñanza e Investigación en Psicología, 21(2), 141-152. https://www.redalyc.org/articulo.oa?id = 29248181004.

Perales G., V., Jiménez R., B. y Sánchez-Aragón, R. (2014). Validación en México de la escala para medir las conductas no verbales de inmediatez afectiva hacia la pareja. En R. Díaz-Loving, S. Rivera-Aragón, I. Reyes-Lagunes y M. M. Flores-Galaz (Eds.): La Psicología Social en México (pp. 627- 632). México: AMEPSO.

Pérez A., G.I., Estrada C., S., Pacheco Q., L.V., García R., L. y Tuz S., M.Á. (2017). Bienestar psicológico y satisfacción sexual en personas de 40 a 70 años de edad. Revista Iberoamericana de las Ciencias Sociales y Humanísticas, 6(11). Doi: 10.23913/ ricsh.v6i11.115.

Pérez P., A. y Sánchez-Aragón, R. (2015, octubre). Eventos hirientes en la relación de pareja (Conferencia). Memorias del XXIII Congreso Mexicano de Psicología. Cancún, Q.R., México.

Rivera-Aragón, S. y Díaz-Loving, R. (2002). La cultura del poder en la pareja. México: Porrúa/UNAM.

Rizo R., P., González R., A., Sánchez C., F., y Murguía M., P. (2015). Tendencia de la mortalidad por cáncer en México: $1990-2012$. Evidencia Médica e Investigación en Salud, 8(1), 5-15. 
Romero P., A., Galicia N., V.V. y Del Castillo A., A. (2017). La satisfacción con la relación de pareja en la actualidad. Educación y Salud. Boletín Cientifico de Ciencias de la Salud del ICSa, 5(10). Doi: 10.29057/icsa.v5i10.2535.

Sánchez-Aragón, R. (1995). El amor y la cercanía en la satisfacción de pareja a través del ciclo de vida. Tesis inédita de doctorado. México: Universidad Nacional Autónoma de México. Recuperado de http://132.248.9.195/pmig2016/0230150/ Index.html.

Sánchez-Aragón, R. (2013). Comunicación emocional, cercanía y satisfacción en las relaciones amorosas. En A. García, J. Wilson y F. Pereira (Eds.): Relacionamento Interpessoal (pp. 173-183). Vitória, Espírito Santo (Brasil): Centro Internacional de Pesquisa do Relacionamento Interpessoal.

Sánchez-Aragón, R. (2018). Afectividad y co-regulación en la pareja: validación de un inventario y cambios a través del ciclo vital. Revista do Psicologia e Educação On-Line, 1(1), 58-69.

Shaver, P., Mikulincer, M., Lavy, S. y Cassidy, J. (2009). Understanding and altering hurt feelings: An attachment-theoretical perspective on the generation and regulation of emotions. En A. Vangelisti (Ed.): Feeling hurt in close relationships (pp. 92-119). Cambridge, UK: Cambridge University Press.

Sociedad Mexicana de Psicología (2010). Código ético del psicólogo. México: Trillas.

Sprung, B.R., Janotha, B.L. y Steckel, A.J. (2011). The lived experience of breast cancer patients and couple distress. Journal of the American Academy of Nurse Practitioners, 23(11), 619-627. Doi: 10.1111/j.1745-7599.2011.00653.x.

Triscoli, C., Croy, I., Olausson, H. y Sailer, U. (2017). Touch between romantic partners: Being stroked is more pleasant than stroking and decelerates heart rate. Physiology \& Behavior, 177(1), 169-175. Doi: 10.1016/j.physbeh.2017.05.006.

Valentine, K.A., Li, N.P., Meltzer, A.L. y Tsai, M.H. (2020). Mate preferences for warmth-trustworthiness predict romantic attraction in the early stages of mate selection and satisfaction in ongoing relationships. Personality and Social Psychology Bulletin, 46(2), 298-311. Doi: 10.1177/0146167219855048.

Vangelisti, A. (2009). Feeling hurt in close relationships. Cambridge, UK: Cambridge University Press.

Vangelisti, A. y Young, S.L. (2000). When words hurt: The effects of perceived intentionality on interpersonal relationships. Journal of Social and Personal Relationships, 17(3), 393-424. Doi: 10.1177/0265407500173005.

Zepeda G., G.D. y Sánchez-Aragón, R. (2019). Efectos del apego, afecto y capacidad de recibir apoyo en la salud de la pareja. Psicologia e Educação On-Line, 2(1), 64-74. 